\title{
Overheating effect and hole-phonon interaction in SiGe heterostructures
}

\author{
I.B. Berkutov, V.V. Andrievskii, and Yu.F. Komnik \\ B. Verkin Institute for Low Temperature Physics and Engineering of the National Academy of Sciences of Ukraine \\ 47 Lenin Ave., Kharkov 61103, Ukraine \\ E-mail: Andrievskii@ilt.kharkov.ua
}

\section{Myronov}

Musashi Institute of Technology, 8-15-1 Todoroki, Setagaya-ku, Tokyo, Japan

\section{O.A. Mironov}

Warwick SEMINANO R\&D Centre, University of Warwick Science Park, Coventry CV4 7EZ, UK

International Laboratory of High Magnetic Fields and Low Temperatures, P.O. Box 4714, 50-985 Wroclaw 47, Poland

Received June 26, 2008

\begin{abstract}
The effect of the charge carriers overheating in a two-dimensional (2D) hole gas in a $\mathrm{Si}_{1-x} \mathrm{Ge}_{x}$ quantum well, where $x=0.13 ; 0.36 ; 0.8,0.95$, has been realized. The Shubnikov-de Haas (SdH) oscillation amplitude was used as a «thermometer» to measure the temperature of overheated holes. The temperature dependence of the hole-phonon relaxation time was found using analysis of dependence of amplitude of SdH oscillations change on temperature and applied electrical field. Analysis of the temperature dependence of the hole-phonon relaxation time exhibits transition of 2D system from regime of «partial inelasticity» to conditions of small angle scattering.
\end{abstract}

PACS: 72.15.Lh Relaxation times and mean free path;

72.20.Ht High-field and nonlinear effects;

72.20.My Galvanomagnetic and other magnetotransport effects.

Keywords: quantum well, 2D hole gas, Shubnikov-de Haas oscillation, hole-phonon relaxation time.

\section{Introduction}

The surface of the crystal experiences surface acoustic waves [1]: Rayleigh and Lamb waves and so on. The interaction of electrons with them differs essentially from the electron-phonon interaction in the bulk of the crystal. The specific features of the electron-phonon interaction are present in thin films of metals and semiconductors because the surface processes more strongly affect the kinetic properties of these objects. In free thin films with $d<\lambda$ ( $d$ is the film thickness, $\lambda$ is the phonon wave length) there are bending waves obeying the quadratic dispersion law $\omega \propto q^{2}$ ( $\omega$ and $q$ are the phonon frequency and momentum, respectively) [2]. Films on a substrate can experience Love waves with shear horizontal polarization. These waves obey an unusual dispersion law $\omega \propto q^{1 / 2}[3]$. The specifics of the phonon spectrum of films affect the character of the electron-phonon interaction. Besides, the space quantization of the electron spec- trum can be an additional factor of influence in semimetallic and semiconducting films. The situation is quite simpler at the interface in semiconducting heterostructures. Here the electron occupies the quantum states in the quantum well (QW) and form a two-dimensional (2D) electron gas, whereas the phonons can be thought of as three-dimensional (3D) since the elastic properties of the crystal are identical at the both sides of the interface. The heterostructures in semiconductors offer a possibility of investigating the electron-phonon interaction between 2D electron gas and 3D phonons.

The aim of this study is to investigate the hole-phonon interaction in a 2D hole gas in SiGe heterostructures, in particular to obtain information about the time and the temperature dependence of the hole-phonon interaction. The use of the quantum corrections to conductivity caused by the effects of weak localization on interaction cannot provide information about the electron-phonon relaxation time at very low temperatures because in this 
condition electron-electron scattering predominates over other inelastic processes. This relaxation time can, however, be found from the electron overheating effect, where the electron temperature $T_{e}$ increases above the phonon temperature $T_{\mathrm{ph}}$ due to a strong electric field (current) or other heating factors. It should be noted that the electron overheating effect in a 2D electron gas is induced by the heat transport through the interface. Estimation of the electron-phonon relaxation time is possible because the transfer of the excess energy from the electron system to the phonon one is controlled by this time, even under the condition of strong elastic scattering. Experimentally, this problem comes to estimating the electron temperature $T_{e}$ which changes under the influence a strong current. To achieve the electron overheating effect, the phonon should be free to leave the conducting layer and come into the crystal surrounding (i.e. good acoustic coupling is required between the conducting layer and the crystal). This requirement is met in heterostructures.

In present study the overheating effect of charge carriers was realized in $p$-type heterostructures with a $\mathrm{Si}_{1-x} \mathrm{Ge}_{x}$ quantum well. The Shubnikov-de Haas oscillation ( $\mathrm{SdHO}$ ) amplitude was used as a «thermometer» to measure the temperature of overheated holes.
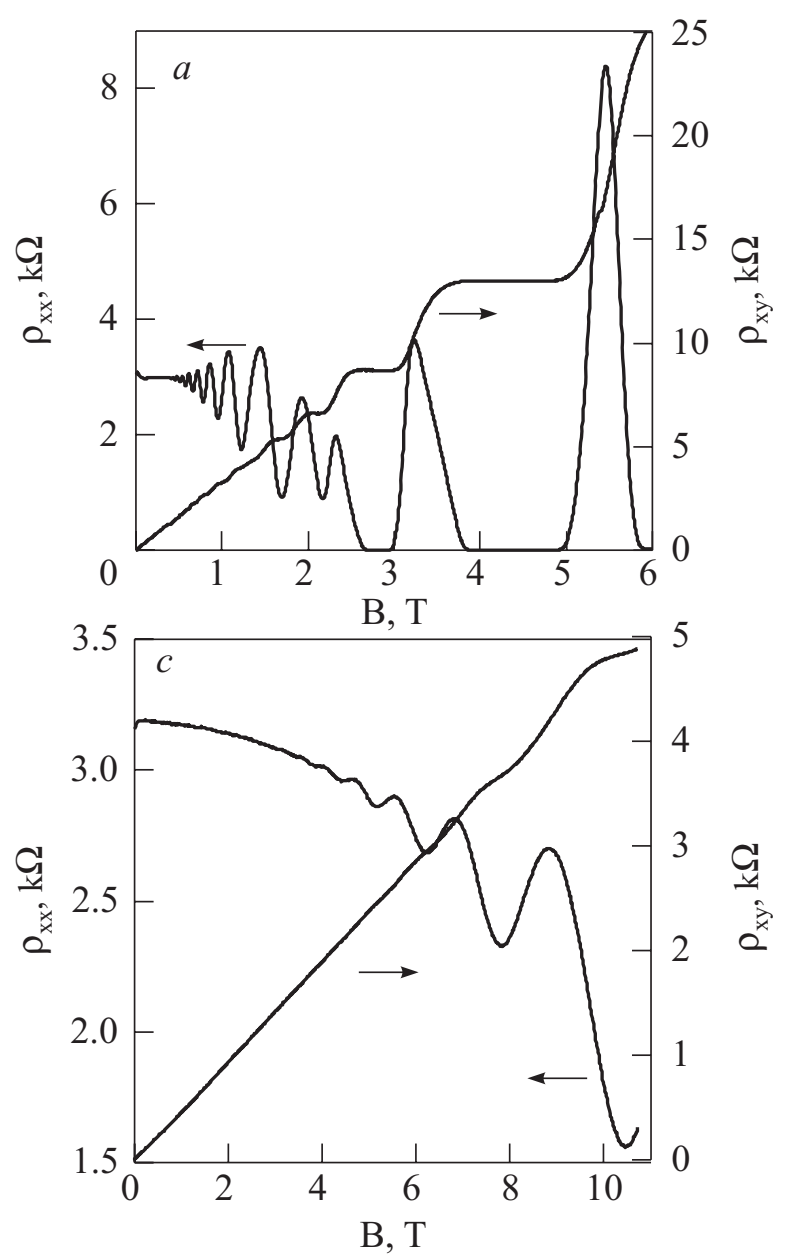

\section{Sample description}

Four of the samples studied in this work (labelled A-D, see Table 1) were prepared by the molecular beam epitaxy technique [4]. In samples A and B the layers are arranged as follows: a single crystal Si $n$-type (100) substrate, pure undoped $\mathrm{Si}$ epitaxial layer, $\mathrm{Si}_{1-x} \mathrm{Ge}_{x}$ quantum well ( $\sim 10 \mathrm{~nm}$ thick), undoped Si spacer $(\sim 20 \mathrm{~nm})$, Si supply layer boron-doped at about $2.5 \cdot 10^{18} \mathrm{~cm}^{-3}$, pure Si cap $(10 \mathrm{~nm})$. In samples $C$ and $D$ the composition of the crystal beneath the QW, the spacer and the boron-doped layers were $\mathrm{Si}_{0.7} \mathrm{Ge}_{0.3}$ and $\mathrm{Si}_{0.37} \mathrm{Ge}_{0.63}$, instead of pure $\mathrm{Si}$.

To measure conductivity Hall bars were prepared, shaped as a «double cross», i.e. a narrow $(\sim 0.5 \mathrm{~mm})$ strip with two pairs of narrow $(0.05 \mathrm{~mm})$ potential bars about $2 \mathrm{~mm}$ apart. The diagonal $R_{x x}$ and off-diagonal $R_{x y}$ components of the resistance tensor were measured as functions of magnetic field on samples $\mathrm{A}$ and $\mathrm{C}$ up to $11 \mathrm{~T}$ and for samples $\mathrm{B}$ and $\mathrm{D}$ up to $6 \mathrm{~T}$. The sample parameters: diagonal resistivity $\rho_{x x}$, hole concentration from Hall $p_{\text {Hall }}$ and SdHO $p_{\mathrm{ShH}}$, mobility $\mu_{\mathrm{Hall}}$ and effective mass $m^{*}$, hole diffusion coefficient $D$ measuring at the lowest temperature are shown in Table 1.
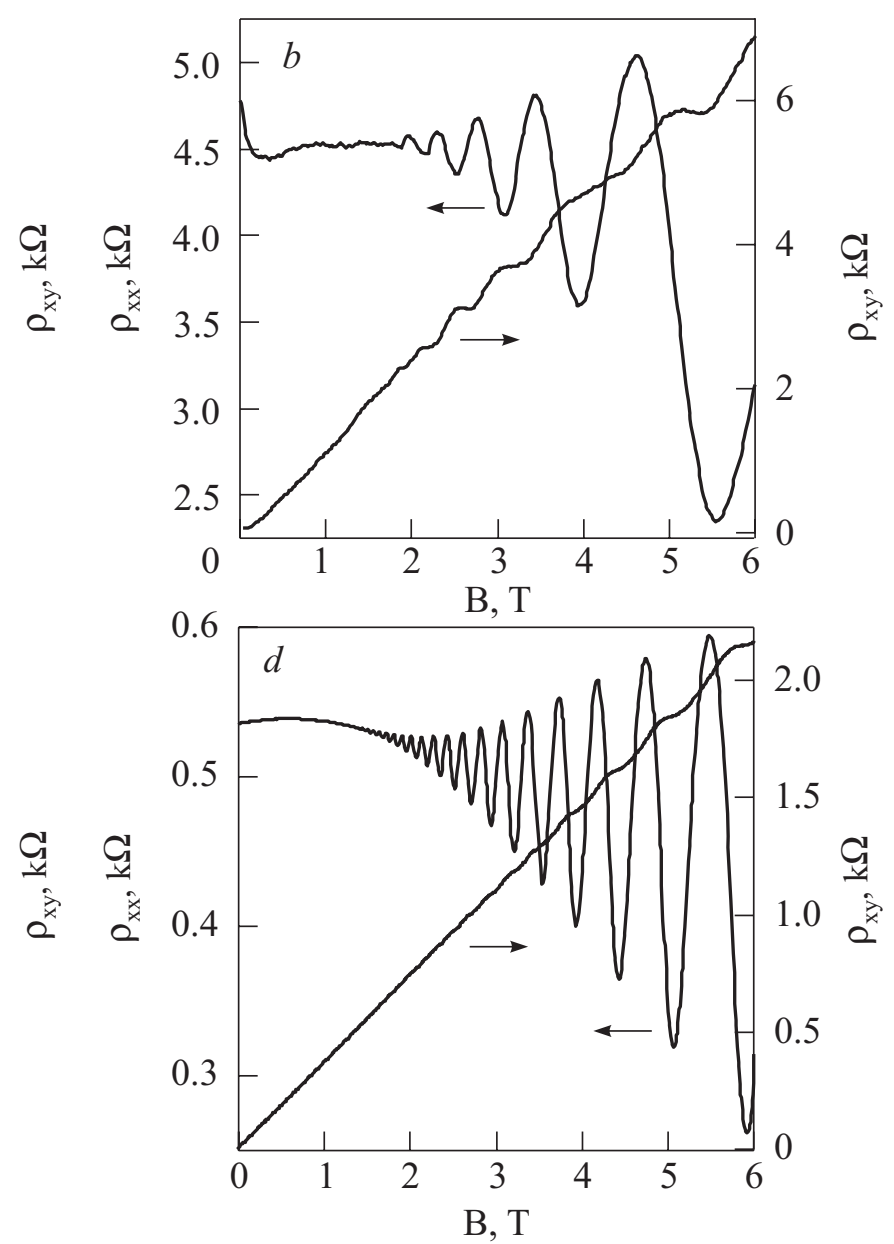

Fig. 1. Magnetoresistance $\rho_{x x}$ and $\rho_{x y}$ of samples A (a), B (b), C (c), D (d). T=33 mK for sample A, and $T \sim 0.3 \mathrm{~K}$ of the other samples. 
Table 1. Characteristic parameters of the samples.

\begin{tabular}{|c|c|c|c|c|c|c|c|}
\hline $\begin{array}{l}\frac{0}{0} \\
\text { 芯 }\end{array}$ & $\begin{array}{l}\overline{\overline{0}} \\
\dot{3} \\
\vdots \\
\vdots \\
\bar{\Xi} \\
\bar{\Xi}\end{array}$ & $\frac{c}{y}$ & 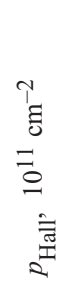 & 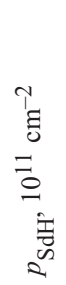 & 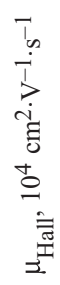 & $\begin{array}{l}\text { O } \\
\Xi \\
\stackrel{*}{\Xi}\end{array}$ & $\begin{array}{l}T_{0} \\
i \\
\text { if } \\
0 \\
0\end{array}$ \\
\hline A & $\mathrm{Si}_{0.87} \mathrm{Ge}_{0.13}$ & 3.04 & 1.89 & 2.0 & 1.16 & 0.24 & 20.7 \\
\hline $\mathrm{B}$ & $\mathrm{Si}_{0.64} \mathrm{Ge}_{0.36}$ & 4.78 & 6.42 & 6.7 & 0.22 & 0,24 & 13.9 \\
\hline $\mathrm{C}$ & $\mathrm{Si}_{0.2} \mathrm{Ge}_{0.8}$ & 3.17 & 15.8 & 14.6 & 0.11 & 0.16 & 29.3 \\
\hline $\mathrm{D}$ & $\mathrm{Si}_{0.05} \mathrm{Ge}_{0.95}$ & 0.54 & 17.5 & 16.2 & 0.68 & 0.156 & 179 \\
\hline
\end{tabular}

The variations in magnetoresistances $\rho_{x x}(B)$ and $\rho_{x y}(B)$ with field, at the lowest temperatures, are illustrated in Fig. 1 ( $\rho$ stands for the resistance per square area of a 2D electron system). The curves exhibit pronounced Shubnikov-de Haas oscillations at $B \geq 1$ T and clear steps of the quantum Hall effect in sample A.

\section{Experimental results and discussion}

Quantum interference effects were used in Ref. 5 to estimate the electron temperature during electron overheating. Electron overheating becomes more obvious when the SdHO are observed [6-8]. In the cited papers the falloff of the amplitude of the oscillations with increasing applied electric field was used to find a relationship between the electron temperature and the rate of loss of excess energy by the electrons. In Ref. 8 the dependence of the energy loss time on the overheating temperature was found, and it was concluded that the main chan- nel of electron energy loss is the emission of acoustic phonons. In the present study, the overheating effect of the charge carriers is used to calculate, straightforwardly, the temperature dependence of the hole-phonon relaxation time in $p$-type $\mathrm{Si}_{1-x} \mathrm{Ge}_{x}$ quantum wells.

In our experiments, the hole temperature $T_{h}$ was found by comparing the $\mathrm{SdHO}$ amplitude change with current and with temperature. As an example, the $\mathrm{SdHO}$ amplitude observed in sample D at low current and varying temperature $(a)$ and at constant temperature and varying current $(b)$ are shown in Fig. 2.

The amplitude variation in these two cases were analyzed for three extrema (with filling factors $v=18,20$, and 22 ) in the magnetic field region $0.8-3.5 \mathrm{~T}$. The quantum numbers vare found from the off-diagonal component of the resistance $\rho_{x y}(B)$ using the equation for resistance quantization under the condition of the quantum Hall effect $\left(h / e^{2} v\right)$.

The electron overheating effect was considered in a number of theoretical studies (their results are applicable to hole overheating as well). In Ref. 9 an expression

$$
T_{e}-T_{\mathrm{ph}}=\frac{E^{2} \sigma}{\gamma T_{e-\mathrm{ph}}} \tau_{e-\mathrm{ph}}\left(T_{e-\mathrm{ph}}\right),
$$

was obtained from the heat balance equation, which contains the characteristic of our interest, i.e. the time of electron-phonon relaxation $\tau_{e-\mathrm{ph}}$ at a certain temperature $T_{e-\mathrm{ph}}$, characterizing the electron-phonon interaction under the electron overheating condition. The prefactor $\gamma$ describes the temperature dependence of the electronic specific heat $C_{e}(T)=\gamma T$. Since this parameter is unknown, it is reasonable to pass on to the equation of Ref. 10

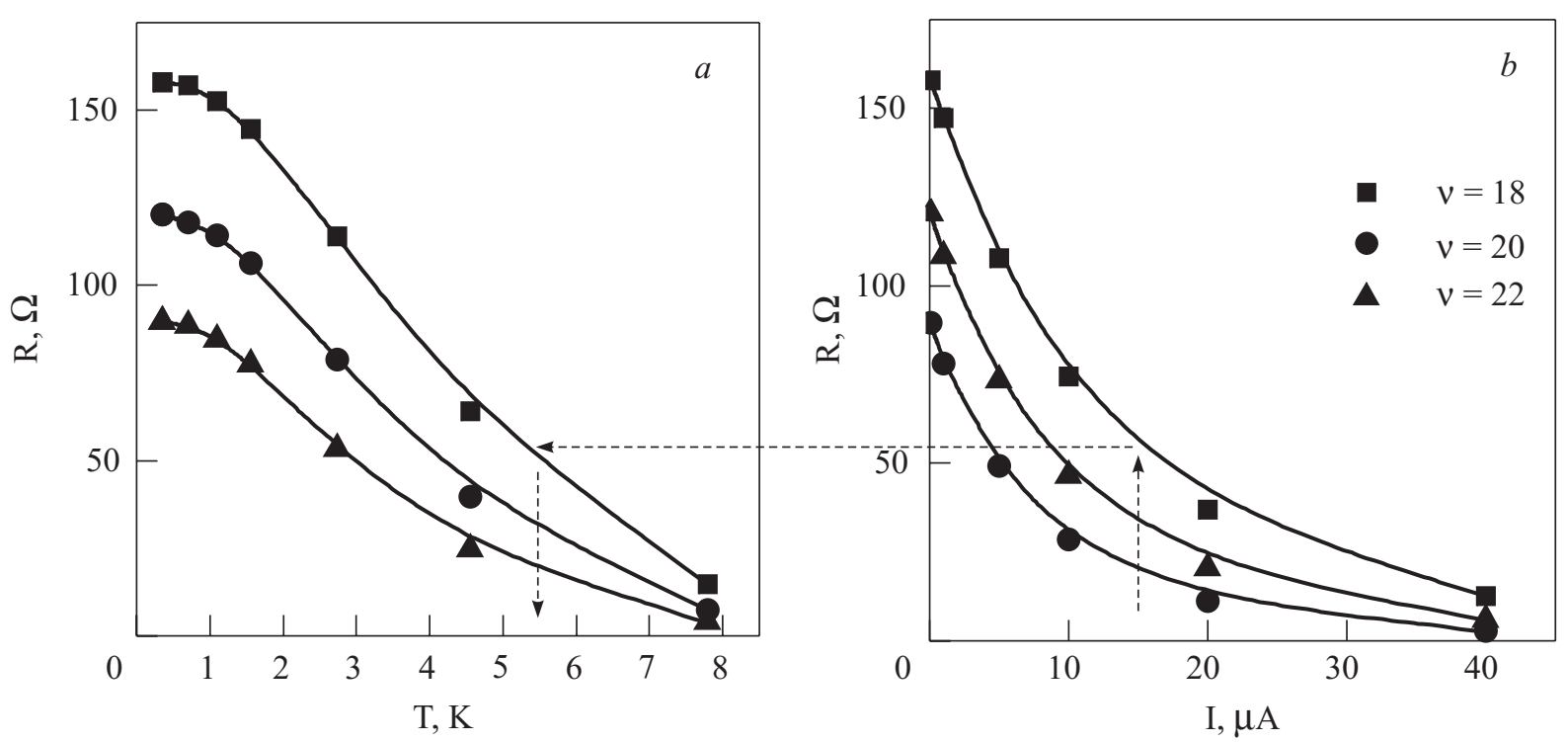

Fig. 2. Dependence of Shubnikov-de Haas oscillation amplitudes for sample D $(a)$ with temperature and $(b)$ with driving current. Solid lines are guides to the eye. 


$$
\left(k T_{e}\right)^{2}=\left(k T_{\mathrm{ph}}\right)^{2}+\left(\frac{6}{\pi^{2}}\right)(e E)^{2} D \tau_{e-\mathrm{ph}} .
$$

This follows from Eq. (1) if we write down the heat capacity and conductivity in terms of density of states $v_{d s}: C_{e}=\left(\pi^{2} / 3\right) k^{2} v_{d s} T$ and $\sigma=e^{2} v_{d s} D$, besides it is necessary to use expressions for $2 \mathrm{D}$ electron gas: $v_{d s}=m^{*} /\left(\pi \hbar^{2}\right), D=(1 / 2) v_{F}^{2} \tau\left(v_{F}\right.$ is the Fermi velocity $v_{F}=\left(\hbar / m^{*}\right)(2 \pi n)^{1 / 2}, n$ is the concentration of $2 \mathrm{D}$ electrons). Equation (2) is quite convenient because it includes only one characteristic of the samples, namely the electron diffusion coefficient $D$. The calculations according to Eq. (2) gives possibility to obtain dependence $\tau_{e-\mathrm{ph}}\left(T_{e-\mathrm{ph}}\right)$. Temperature $T_{e-\mathrm{ph}}$ is taken to be the mean $T_{e-\mathrm{ph}}=\left(T_{\mathrm{ph}}+T_{e}\right) / 2[11,12]$, here $T_{\mathrm{ph}}$ corresponds to the temperature of the crystal.

The temperature dependences of the hole-phonon relaxation time $\tau_{h-\mathrm{ph}}$ of all the samples are shown in Fig. 3. The dependences $\tau_{h-\mathrm{ph}}\left(T_{h-\mathrm{ph}}\right)$ above $1 \mathrm{~K}$ for sample D and above $\sim 0.5-0.6 \mathrm{~K}$ for the other samples can be approximated by the power function $\tau_{e-\mathrm{ph}}^{-1}=9 \cdot 10^{-9} T^{2}$ (Fig. 3, solid lines). It is essential that this function is common despite the different characteristics of the samples. Moreover, the points corresponding to the filling factors $v$ different for each sample fall on the same curve, which suggests that the $\tau_{h-\mathrm{ph}}$ - value is independent of the magnetic field. A stronger dependence $\tau_{h-\mathrm{ph}}\left(T_{h-\mathrm{ph}}\right)$ appears below the temperatures specified above.

The results obtained must be interpreted in the contexts of the theoretical studies considering the temperature variations of electron-phonon relaxation for 2D electrons interacting with $3 \mathrm{D}$ phonons $[13,14]$. In Ref. 14

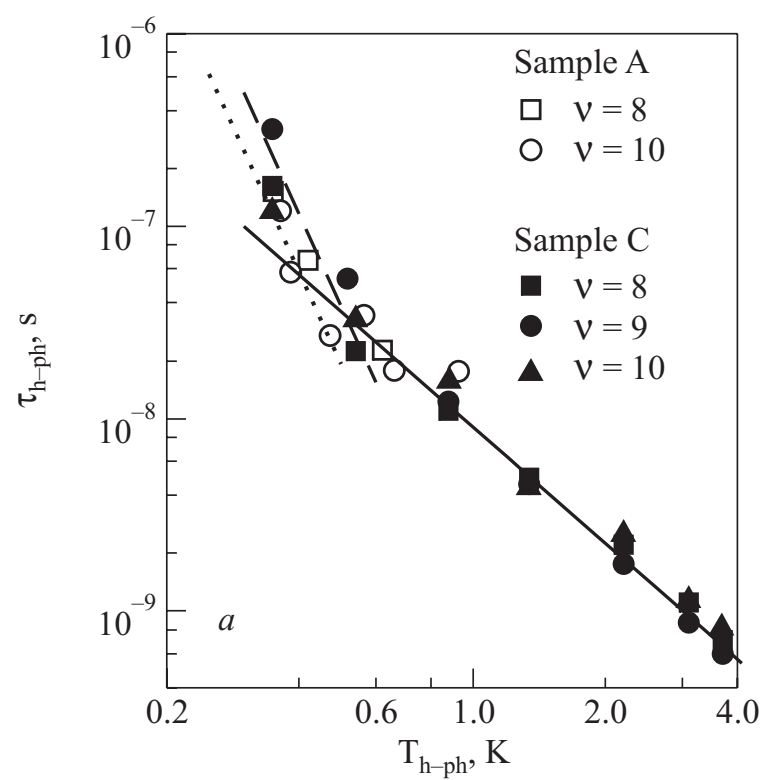

these variations are analyzed in a wide range of temperatures. The energy is quantized in a $\mathrm{QW}$ :

$$
E_{1}=\frac{p_{x}^{2}}{2 m_{x}^{*}}+\frac{p_{y}^{2}}{2 m_{y}^{*}}+\frac{\pi^{2} \hbar^{2}}{2 m_{z}^{*} L^{2}} n^{2},
$$

where $L$ is the width of the well, $n$ is the quantum number. Electrons occupied the ground state in the QW with the energy $E_{1}=\pi^{2} \hbar^{2} / 2 m_{z}^{*} L^{2}$. The electron-phonon interaction of $2 \mathrm{D}$ carriers is limited on variation of the transverse momentum of the electrons in direction of quantization (in $z$ direction). The transverse component of the momenta of the emitted (absorbed) phonons is determined by the width of the QW $q_{\perp} \sim 2 \pi / L$. At high temperatures the thermal phonon momentum is $q_{T}>2 \pi / L$, and the electron-phonon scattering is accompanied by emission (absorption) of phonons with the wave vector mainly perpendicular of the QW. The process is characterized by the dependence $\tau_{e-\mathrm{ph}}^{-1} \propto T$. At lower temperatures the momentum of the thermal phonon $q_{T}=k_{B} T / \hbar s$ ( $s$ is the sound velocity) is smaller than $2 \pi / L$. But if the phonon is capable to change the wave vector of the electron by the maximum value $2 k_{F}$, the energy exchange between the electron and the lattice can be about $k_{B} T$. We expect the dependence $\tau_{e-\mathrm{ph}}^{-1} \propto T^{2}$ in this region of «partial inelasticity». At even lower temperatures $\left(q_{T}<2 k_{F}\right)$ the wave vector of the emitted (absorbed) phonon is limited by temperature and the scattering is similar to the small-angle scattering in a 3D metal following the dependence $\tau_{e-\mathrm{ph}}^{-1} \propto T^{5}$.

The obtained dependencies $\tau_{h-\mathrm{ph}}^{-1} \propto T^{2}$ (Fig. 3) correspond to the region of «partial inelasticity» [14]. At lowering temperatures the dependence becomes stronger. We

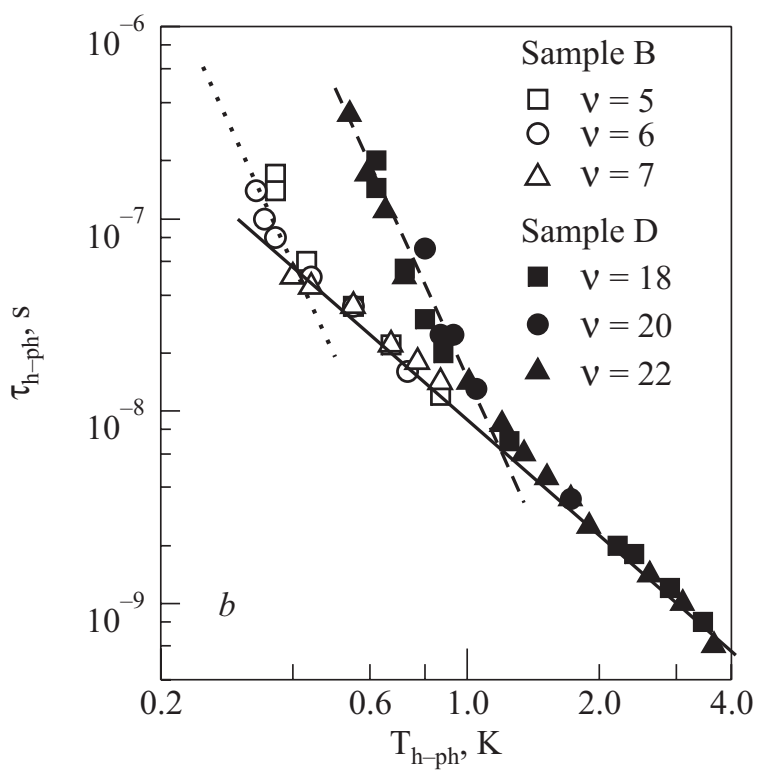

Fig. 3. Temperature dependence of hole-phonon relaxation times $\tau_{h-p h}$ found from the overheating effect. Solid line is dependence $\tau_{e-\mathrm{ph}}^{-1}=9 \cdot 10^{-9} T^{2}$, doted line is dependence $\tau_{e-\mathrm{ph}}^{-1}=0.6 \cdot 10^{-9} T^{5}$, dash line is dependence $\tau_{e-\mathrm{ph}}^{-1}=1.2 \cdot 10^{-9} T^{5}$, short dash line is dependence $\tau_{e-\mathrm{ph}}^{-1}=1.5 \cdot 10^{-8} T^{5}$. 
attribute this to the small-angle mechanism predict in theory [14], which is characterized by the dependence $\tau_{e-\mathrm{ph}}^{-1} \propto T^{5}$. The temperature of the transition to this dependence obeys qualitatively (to within the numerical coefficient $<<1$ ) the condition $q_{T} \sim 2 k_{F}$, if we take $k_{F}=(2 \pi n)^{1 / 2}$ for a $2 \mathrm{D}$ electron gas, i.e., the temperature grows as the carrier concentration increases. The quantitative discrepancy is not clear yet and may be connected with conditional character of the formulas for 2D electron gas to the real situation.

1. I.A. Viktorov, Rayleigh and Lamb Waves: Physical and Application, New York, Plenum Press (1967).

2. I.M. Lifshits, Zh. Eksp. Teor. Fiz. 22, 471 (in Russian) (1952).

3. E.S. Syrkin, Yu.F. Komnik, and E.Yu. Beliaev, Low Temp. Phys. 22, 80 (1996).

4. T.J. Grasly, C.P. Parry, P.J. Phillips, B.M. McGregor, R.J.H. Morris, G. Braithwaite, T.E. Whall, E.H.C. Parker, R. Hammond, A.P. Knights, and P.G. Coleman, Appl. Phys. Lett. 74, 1848 (1999).
5. R. Fletcher, J.J. Harris, C.T. Foxon, and R. Stoner, Phys. Rev. B45, 6659 (1992).

6. P.T. Coleridge, R. Stoner, and R. Fletcher, Phys. Rev. B39, 1120 (1989).

7. K.J. Lee, J.J. Harris, A.J. Kent, T. Wang, S. Sakai, D.K. Maude, and J.C. Portal, Appl. Phys. Lett. 78, 2893 (2001).

8. G. Stöger, G. Brunthaler, G. Bauer, K. Ismail, B.S. Meyerson, J. Lutz, and F. Kuchar, Semicond. Sci. Technol. 9, 765 (1994).

9. P.W. Anderson, E. Abrahams, and T.W. Ramakrishnan, Phys. Rev. Lett. 43, 718 (1979).

10. S. Hershfield and V. Ambegaokar, Phys. Rev. B34, 2147 (1986).

11. S.I. Dorozhkin, F. Lell, and W. Schoepe, Solid State Commun. 60, 245 (1986).

12. V.V. Andrievskii, I.B. Berkutov, Yu.F. Komnik, O.A. Mironov, and T.E. Whall, Fiz. Nizk. Temp. 26, 1202 (2000) [Low Temp. Phys. 26, 890 (2000)].

13. P.J. Price, Ann. Phys. 133, 217 (1988).

14. V. Karpus, Sov. Phys. Semicond. 20, 6 (1986). 